\title{
MITIGATION OF WAVE LOADS ON THE FLOATING POROUS STRUCTURE BY SLOTTED SCREENS
}

\author{
KOTTALA PANDURANGA \& SANTANU KOLEY \\ Department of Mathematics, Birla Institute of Technology and Science - Pilani, Hyderabad Campus, India
}

\begin{abstract}
This paper presents the effectiveness of partially immersed slotted screens placed at a finite distance away from the floating porous breakwater to mitigate the wave-induced hydrodynamic forces on the floating breakwater. The fluid motion within the porous structure is analyzed using the Sollitt and Cross model. Further, a non-linear pressure drop condition on the slotted screens is considered, which includes the effects of wave height on the incident wave energy dissipation by the slotted screens. In the presence of the non-linear boundary condition on the slotted screen, the associated boundary value problem is solved numerically using the iterative multi-domain boundary element method. Various physical interests of the study, such as reflection coefficient, transmission coefficient, wave energy dissipation coefficient, and wave forces acting on the slotted screens and floating breakwater, are analyzed in detail for various wave and structural parameters. The results show that the maximum wave-induced forces acting on the porous breakwater are observed when the gap between the slotted screen and porous breakwater is approximately equal to an integer multiple of half of the wavelength for different porosities of the slotted screens.

Keywords: slotted screens, wave-induced forces, iterative multi-domain boundary element method, reflection coefficient.
\end{abstract}

\section{INTRODUCTION}

Floating porous structures are commonly used as effective wave energy attenuators in the coastal environment to protect the harbours and seawall structures from wave attacks. Due to the continuous wave action on the floating porous structures and the demand of the serviceability requirements, it is necessary to mitigate the wave-induced hydrodynamic forces acting on the structures.

There are several ways to mitigate the wave-induced forces and structural responses of floating structures. One among them is the installation of the bottom-founded breakwater (Wang et al. [1]). However, the building of such conventional breakwaters is inadequate in reducing the structural response of floating structures as they prevent the water circulation around the structure, leading to environmental damage (Hong et al. [2]). Moreover, the construction of the conventional breakwaters is complex and more expensive as the depth of the sea increases. Therefore, several researchers proposed alternative wave attenuating structures that could effectively mitigate the wave forces and work as an anti-motion device for the floating structure such as the floating breakwater (Tay et al. [3]), mooring lines (Nguyen et al. [4]), horizontal and vertical anti-motion plates attached to the structures (Ohta [5]), submerged horizontal and inclined porous plates (Watanabe et al. [6], Cheng et al. [7]), gill cells with the perforated bottom surface (Wang et al. [8]), etc. A semi-rigid connector in reducing the structural response of the interconnected beams was used by Wang et al. [9]. Further, the study was extended by Gao et al. [10] using a flexible hinge-line connector. It was observed that a flexible hinge-line connector was found to be more effective in reducing the structural deflection of the floating VLFS than a semi-rigid connector when the incident wavelength is small. Further, installing an anti-motion device such as OWC-WEC (oscillating water column wave energy converter device) in front of the floating VLFS can also effectively decreases the hydroelastic responses of floating VLFSs. Hong et al. [11] 
proposed an analytical method to evaluate the structural reduction efficiency of a T-shaped freely floating breakwater with a built-in OWC device.

The aforementioned anti-motion systems can also be used to reduce wave-induced hydrodynamic forces on the floating breakwater, in addition to mitigating wave-induced structural responses. Huang et al. [12] presented a detailed review on the wave reflection and transmission characteristics of perforated/slotted screens, as well as the incident wave energy dissipation mechanism by perforated/slotted vertical and horizontal screens, and the reduction of wave-induced forces acting on the bottom standing structures by surfacepiercing floating perforated/slotted screens. Vijay et al. [13] studied the effectiveness of pair of permeable plates on reducing structural responses and wave forces on the floating structure using the multi-domain boundary element method. Their study revealed that the floating structure experiences the minimum wave forces when the twin porous plates are positioned in the middle of the floating structure and the rigid wall. Sun et al. [14] examined the installation of a submerged porous breakwater to mitigate wave loads on the floating bridge decks. It was seen that the construction of the submerged porous breakwater at suitable locations could significantly reduce the horizontal wave loads on the floating structure. Using the method of matched eigenfunction expansions, Qiao et al. [15] studied the motion responses and wave forces on the floating rigid breakwater attached to a pair of perforated side plates. The wave-induced hydrodynamic forces on the floating rigid body reduced significantly with an increase in the porous-effect parameter of the sides-walls when the breakwater system is rotated about the center (lies at the bottom) of the floating body. From these studies, it is observed that the slotted screens have a great impact on mitigating the wave-induced loads on floating bodies. Further, several researchers considered the linearized pressure drop condition across the perforated/slotted breakwater using the Darcy's law (Qiao et al. [15], Yip and Chwang [16]). But in general, the flow separation through the slotted screen is quadratic in nature. The significance of this quadratic pressure drop condition is that it includes the effects of wave height in dissipating the incident wave energy (Liu and Li [17]).

In the present work, the mitigation of wave forces on the rectangular-shaped floating porous breakwater by placing a pair of slotted screens on either side of the floating porous breakwater is studied using the linear potential flow theory. The fluid motion in the porous breakwater is analyzed using the Sollitt and Cross model [18]. Further, a non-linear (quadratic) pressure drop condition on the slotted screen is adopted to study the efficiency of the slotted screens on mitigating the wave loads acting on the floating porous breakwater. An iterative boundary element method (BEM) is adopted to handle the non-linear pressure drop condition on the slotted screen.

\section{MATHEMATICAL FORMULATION}

The schematic geometry of the rectangular-shaped floating porous breakwater protected by two slotted screens placed on either side of the breakwater is shown in Fig. 1. To study the interaction of the regular waves with the floating porous breakwater system, a twodimensional Cartesian system is adopted where the $x$-axis is taken as the horizontal axis, and $Z$-axis is taken vertically upward. Consider linear small-amplitude simple harmonic water waves of wave height $H$, wavelength $L$, and circular frequency $\omega$ propagating along with the positive $x$-axis. For mathematical description, the structural parameters are represented as water depth $h$, submergence depth of the porous breakwater $d_{2}$, the width of the porous breakwater $B$, submergence depth of the slotted screens $d_{1}$. The space between the slotted screen and breakwater is $w$ (on either side of the breakwater). In the presence of the breakwater system and pair of slotted screens, the total fluid domain is divided into six 
regions $\Omega_{j}, j=1,2, \ldots 6$, as shown in Fig. 1. The computational domain of the present physical problem is shown in Fig. 2.

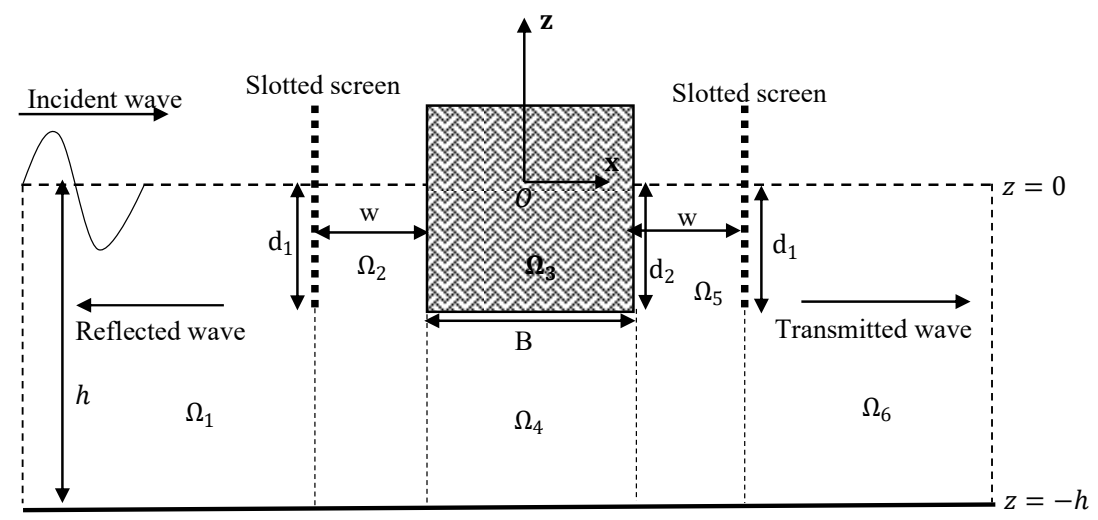

Figure 1: Schematic diagram of floating porous breakwater protected by pair of slotted screens.

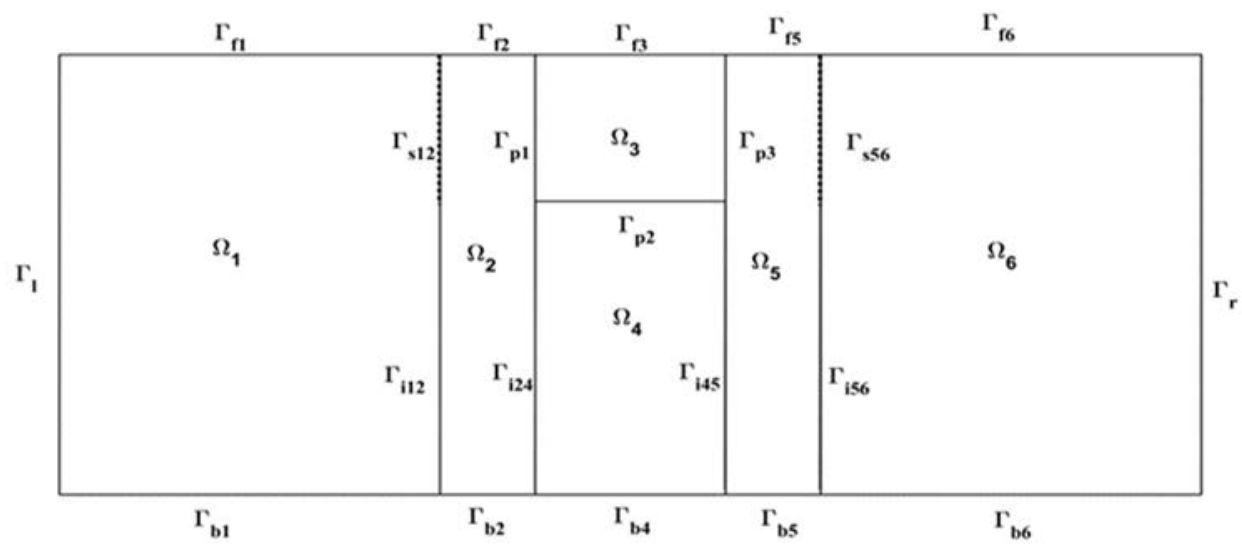

Figure 2: Computational domain of the physical problem.

It is assumed that the linear potential flow theory applies, i.e., the flow is incompressible, inviscid, and its motion is irrotational. Then, the velocity potential $\Phi_{j}(x, z, t)$ in every region is expressed as $\Phi(x, z, t)=\operatorname{Re}\left\{\phi(x, z) e^{-\mathrm{i} \omega t}\right\}$, where the spatial velocity potentials satisfy the Laplace equation

$$
\frac{\partial^{2} \phi^{(j)}}{\partial x^{2}}+\frac{\partial^{2} \phi^{(j)}}{\partial z^{2}}=0, \quad j=1,2, \ldots 6
$$

where the superscript $j$ corresponds to the regions $j$ for $j=1,2, \ldots 6$.

The velocity potentials $\phi^{(j)}$ also satisfy the following boundary conditions (BC) 
1. The linearized free-surface $\mathrm{BC}$ at $z=0$ is given by

$$
\frac{\partial \phi^{(j)}}{\partial z}-\frac{\omega^{2}}{g}(s+\mathrm{i} f) \phi^{(j)}=0, \text { on } \quad \Gamma_{\mathrm{f} j} \text { for } j=1,2,3,5,6,
$$

where $\mathrm{s}$ is the inertial coefficient and $\mathrm{f}$ is the friction coefficient of the porous region. It is to be noted that $s=1, f=0$ should be taken for the open water regions.

2. The interface BCs between the regions $\Omega_{1}, \Omega_{2}, \Omega_{4}, \Omega_{5}$, and $\Omega_{6}$ are written as

$$
\begin{gathered}
\phi^{(1)}=\phi^{(2)}, \quad \frac{\partial \phi^{(1)}}{\partial x}=\frac{\partial \phi^{(2)}}{\partial x}, \text { on } \Gamma_{\mathrm{i} 12}, \phi^{(2)}=\phi^{(4)}, \frac{\partial \phi^{(2)}}{\partial x}=\frac{\partial \phi^{(4)}}{\partial x}, \text { on } \Gamma_{\mathrm{i} 24,} \\
\phi^{(4)}=\phi^{(5)}, \frac{\partial \phi^{(4)}}{\partial x}=\frac{\partial \phi^{(5)}}{\partial x}, \text { on } \Gamma_{\mathrm{i} 45}, \phi^{(5)}=\phi^{(6)}, \frac{\partial \phi^{(5)}}{\partial x}=\frac{\partial \phi^{(6)}}{\partial x}, \text { on } \Gamma_{\mathrm{i} 56} .
\end{gathered}
$$

3. The $\mathrm{BC}$ on the horizontal seabed is written as

$$
\frac{\partial \phi^{(j)}}{\partial z}=0, \quad \text { on } \quad \Gamma_{\mathrm{b} j} \text { for } j=1,2,4,5,6
$$

4. The interface BCs between the open water regions $\Omega_{2}, \Omega_{5}$, and porous regions $\Omega_{3}$, $\Omega_{4}$ are given by [18]

$$
\left\{\begin{array}{l}
\phi^{(2)}=(s+\mathrm{i} f) \phi^{(3)}, \frac{\partial \phi^{(2)}}{\partial x}=\epsilon \frac{\partial \phi^{(3)}}{\partial x}, \text { on } \Gamma_{p 1} \\
\phi^{(4)}=(s+\mathrm{i} f) \phi^{(3)}, \frac{\partial \phi^{(4)}}{\partial z}=\epsilon \frac{\partial \phi^{(3)}}{\partial z}, \text { on } \Gamma_{p 2} \\
\phi^{(5)}=(s+\mathrm{i} f) \phi^{(3)}, \frac{\partial \phi^{(5)}}{\partial x}=\epsilon \frac{\partial \phi^{(3)}}{\partial x}, \text { on } \Gamma_{p 3}
\end{array}\right.
$$

5. The non-linear (quadratic) pressure drop condition and the continuity of horizontal fluid velocity at the slotted screen is given by [17]

$$
\begin{aligned}
& \left\{\begin{array}{c}
\phi^{(1)}-\phi^{(2)}=-\frac{8 i}{3 \pi \omega} \frac{1-\tau_{1}}{2 \mu \tau_{1}^{2}}\left|\frac{\partial \phi^{(1)}}{\partial x}\right| \frac{\partial \phi^{(1)}}{\partial x}-2 C_{1} \frac{\partial \phi^{(1)}}{\partial x}, \\
\frac{\partial \phi^{(1)}}{\partial x}=\frac{\partial \phi^{(2)}}{\partial x},
\end{array}\right. \\
& \left\{\begin{array}{cc}
\phi^{(5)}-\phi^{(6)}=-\frac{8 i}{3 \pi \omega} \frac{1-\tau_{2}}{2 \mu \tau_{2}^{2}}\left|\frac{\partial \phi^{(5)}}{\partial x}\right| \frac{\partial \phi^{(5)}}{\partial x}-2 C_{2} \frac{\partial \phi^{(5)}}{\partial x} & \text { on } \Gamma_{s 56,}, \\
\frac{\partial \phi^{(5)}}{\partial x}=\frac{\partial \phi^{(6)}}{\partial x}, &
\end{array}\right.
\end{aligned}
$$


where $\tau_{j}(j=1,2)$ are the porosity (geometrical) of the slotted screens, and $\mu$ is the discharge coefficient, respectively. Further, the expressions for the blockage coefficients $C_{j}$ are given by [17]

$$
C_{j}=0.5 d\left(\frac{1}{\tau_{j}}-1\right)+\frac{\delta}{\pi}\left\{1-\ln \tau_{j}+\frac{1}{3} \tau_{j}^{2}+\frac{281}{180} \tau_{j}^{4}\right\}, \quad j=1,2
$$

with $d$ being the thickness of the screen and $\delta$ being the distance between the centers of two adjacent slots.

6. The far-field BCs at the two auxiliary boundaries placed at $x=-l$ and $x=r$ are represented as

$$
\begin{gathered}
\lim _{x \rightarrow-\infty}\left(\frac{\partial\left(\phi^{(1)}-\phi^{(0)}\right)}{\partial x}+\mathrm{i} k_{0}\left(\phi^{(1)}-\phi^{(0)}\right)\right)=0, \\
\lim _{x \rightarrow \infty}\left(\frac{\partial \phi^{(6)}}{\partial x}-\mathrm{i} k_{0} \phi^{(6)}\right)=0,
\end{gathered}
$$

where $\phi^{(0)}=-\frac{i g H}{2 \omega} e^{i k_{0} x} Z_{0}(z), Z_{0}(z)=\frac{\cosh k_{0}(z+h)}{\cosh k_{0} h}$, and $k_{0}$ is the real positive root of the dispersion relation $\omega^{2}=g k \tanh k h$.

\section{ITERATIVE BOUNDARY ELEMENT METHOD}

In this section, an iterative boundary element method is adopted to solve the associated boundary value problem, as discussed in Section 2. Applying Green's second identity to the velocity potential $\phi$ and Green's function $G$, we obtain the resulting integral equation in each of the regions $\Omega_{j}$ for $j=1,2, \ldots 6$, as ([20])

$$
\frac{1}{2} \phi^{(j)}(\xi, \eta)=\int_{\Gamma}\left(\phi^{(j)} \frac{\partial G^{(j)}}{\partial n_{j}}-G^{(j)} \frac{\partial \phi^{(j)}}{\partial n_{j}}\right) d \Gamma
$$

where $n_{j}$ represents the unit outward normal to the boundary $\Gamma_{j}$ of region $j$, and the Green's function and its normal derivatives are given by

$$
G^{(j)}=\frac{1}{2 \pi} \ln r, \frac{\partial G^{(j)}}{\partial n}=\frac{1}{2 \pi r} \frac{\partial r}{\partial n}, r=\sqrt{(x-\xi)^{2}+(z-\eta)^{2}}
$$

In eqn $(11),(x, z)$ and $(\xi, \eta)$ are the field and source points, respectively. Now, discretize the boundaries of each of the regions $\Omega_{j}, j=1,2, \ldots, 6$, into $N_{j}$ (number of boundary elements on the boundary $\Gamma_{j}$ of the region $\Omega_{j}$ ) smooth elements. It is assumed that on each boundary element, $\phi^{(j)}$ and $\partial \phi^{(j)} / \partial n$ are constants. The discretized form of eqn (10) can be written as [20], [21]

$$
\left[\alpha_{m n}^{(j)}\right]\left\{\phi_{n}^{(j)}\right\}+\left[\beta_{m n}^{(j)}\right]\left\{\frac{\partial \phi_{n}^{(j)}}{\partial n_{j}}\right\}=0
$$


for $m, n=1,2,3, \ldots, N_{j}, j=1,2, \ldots, 6$, in which the influence coefficients $\alpha_{m n}^{(j)}$ and $\beta_{m n}^{(j)}$ are expressed as

$$
\alpha_{m n}^{(j)}=-\frac{1}{2} \delta_{m n}+\int_{\Gamma_{j, n}} \frac{\partial G^{(j)}}{\partial n_{j}} d \Gamma, \quad \beta_{m n}^{(j)}=\int_{\Gamma_{j, n}} G^{(j)} d \Gamma
$$

In eqn (12), $\phi_{n}^{(j)}$ and $\frac{\partial \phi_{n}^{(j)}}{\partial n_{j}}$ denote the velocity potential and its normal derivative at the midpoint of the $n^{\text {th }}$ boundary element of $j^{\text {th }}$ region. Further, $\delta_{m n}$ represents the Kronecker delta. On the other hand, the slotted screens share the same elements, i.e., the first (left) slotted screen shares regions 1 and 2, similarly the second (right) slotted screen shares the regions 5 and 6 . Therefore, on the first slotted screen, i.e., on $\Gamma_{s 12}$, the $m^{\text {th }}$ boundary element of region 1 and $n^{\text {th }}$ boundary element of region 2 is the same. The pressure drop condition on the boundary $\Gamma_{s 12}$ is discretized as [17]

$$
\phi_{n}^{(2)}=\phi_{m}^{(1)}+Z_{1 m} \frac{\partial \phi_{m}^{(1)}}{\partial n_{1}}
$$

where

$$
Z_{1 m}=\frac{8 i}{3 \pi \omega} \frac{1-\tau_{1}}{2 \mu \tau_{1}^{2}}\left|\frac{\partial \phi_{m}^{(1)}}{\partial n_{1}}\right|+2 C_{1}
$$

Further, the discretized form of the continuity of horizontal velocity is written as

$$
\frac{\partial \phi_{n}^{(2)}}{\partial n_{2}}=-\frac{\partial \phi_{m}^{(1)}}{\partial n_{1}}
$$

Similarly, the boundary conditions on the second slotted screen, i.e., on $\Gamma_{s 56}$ are discretized as

$$
\begin{gathered}
\frac{\partial \phi_{n}^{(6)}}{\partial n_{6}}=-\frac{\partial \phi_{m}^{(5)}}{\partial n_{5}}, \\
\phi_{n}^{(6)}=\phi_{m}^{(5)}+Z_{1 m} \frac{\partial \phi_{m}^{(5)}}{\partial n_{5}},
\end{gathered}
$$

where

$$
Z_{2 m}=\frac{8 i}{3 \pi \omega} \frac{1-\tau_{2}}{2 \mu \tau_{2}^{2}}\left|\frac{\partial \phi_{m}^{(5)}}{\partial n_{5}}\right|+2 C_{2}
$$

In a similar manner, all other boundary conditions are discretized. Now, substitute all the discretized BCs along with eqns (14)-(19) into eqn (12) to obtain $\phi^{(j)}$ and $\partial \phi^{(j)} / \partial n$ over all boundary elements of each of the regions. It is to be noted that eqns (14) and (18) are nonlinear. Thus, an iterative procedure is needed to evaluate them. The steps for the iterative procedure are as follows. 
Step 1: Set $\frac{\partial \phi_{m}^{(1)}}{\partial n_{1}}$ and $\frac{\partial \phi_{m}^{(5)}}{\partial n_{5}}$ equal to zero (initial guess) in eqns (14) and (18) for the initial iteration, and determine $Z_{1 m}$ and $Z_{2 m}$. Then the algebraic system (12) becomes linear.

Step 2: Solve the algebraic system (12) using the Gauss elimination method to get the updated values of $\frac{\partial \phi_{m}^{(1)}}{\partial n_{1}}$ and $\frac{\partial \phi_{m}^{(5)}}{\partial n_{5}}$.

Step 3: If the difference between the initial and updated values of $\frac{\partial \phi_{m}^{(1)}}{\partial n_{1}}$ and $\frac{\partial \phi_{m}^{(5)}}{\partial n_{5}}$ lies within the required error limit (i.e., $10^{-4}$ ), stop at step 2. Otherwise, take the average values updated $\frac{\partial \phi_{m}^{(1)}}{\partial n_{1}}$ and $\frac{\partial \phi_{m}^{(5)}}{\partial n_{5}}$ and set it as a new initial guess and repeat from Step 2. In the present analysis, the required accuracy is obtained for not more than 20 iterations. Once $\phi_{n}^{(j)}$ and $\frac{\partial \phi_{n}^{(j)}}{\partial n_{j}}$ are obtained over each boundary element, the reflection coefficient $C_{R}$ and transmission coefficient $C_{T}$ are obtained by

$$
\begin{gathered}
C_{R}=\left|-1+\frac{1}{N_{0}^{2}} \frac{2 i \omega}{g H} e^{i k_{0} l} \int_{-h}^{0} \phi^{(1)}(-l, z) Z_{0}(z) d z\right|, \\
C_{T}=\left|\frac{1}{N_{0}^{2}} \frac{2 i \omega}{g H} \int_{-h}^{0} \phi^{(6)}(r, z) Z_{0}(z) d z\right|,
\end{gathered}
$$

where $N_{0}^{2}=\int_{-h}^{0} Z_{0}^{2}(z) d z$. The energy loss coefficient $C_{L}$ is defined as (see [22] for detailed derivations)

$$
C_{L}=\left(1-C_{R}^{2}-C_{T}^{2}\right)
$$

The dimensionless horizontal and vertical wave forces acting on the porous floating breakwater are evaluated using the following.

$$
F_{x}=\left|\frac{\omega}{g h^{2}}(s+\mathrm{i} f) \int_{\Gamma_{p 1}} \phi^{(3)} n_{x} d \Gamma\right|, \quad F_{z}=\left|\frac{\omega}{g h^{2}}(s+\mathrm{i} f) \int_{\Gamma_{p 2}} \phi^{(3)} n_{z} d \Gamma\right| .
$$

\section{VALIDATIONS}

In Fig. 3(a) and 3(b), the present iterative-BEM-based solutions are compared with the analytical solutions of Hu et al. [23] and Zhu and Chwang [19]. In Fig. 3(a), the reflection coefficient $C_{R}$ and the transmission coefficient $C_{T}$ are plotted against the non-dimensional wave number $k_{0} d_{2}$ with the physical parameters $d_{2}=1$ and $h=6.0 d_{2}$ for a floating rigid structure without slotted screens. It is well known that the porous structure and slotted screens become rigid when $\epsilon=0.0, s=1.0, f=0.0$ and $\tau_{j}=0.0, j=1,2$, respectively. On the other hand, the slotted screen will disappear when the porosity of the screen is set to unity. As a limiting case to compare the present numerical solutions with Zhu and Chwang's [19] theoretical solutions, the floating rigid structure, and the first slotted screen with $\tau_{1}=0.5$ are fully extended till the bottom seabed with $h=8 \mathrm{~m}$. The comparisons reveal that there a good agreement between the present iterative-BEM solutions and theoretical results. 


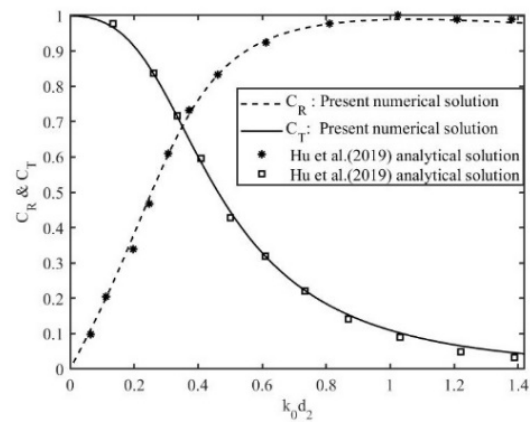

(a)

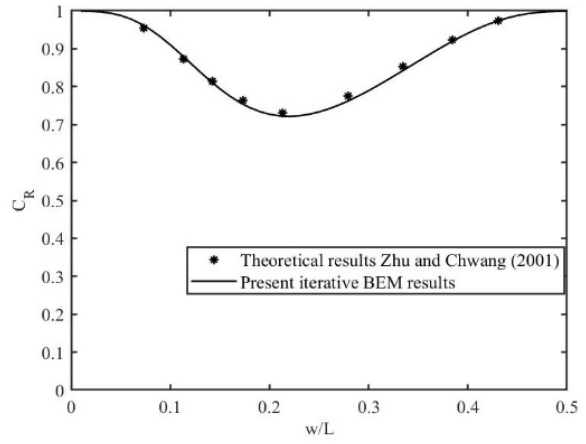

(b)

Figure 3: Comparison of present numerical solution with (a) Hu et al. [23]; and (b) Zhu and Chwang [19] theoretical solutions.

\section{RESULTS AND DISCUSSIONS}

Here, some of the numerical results are presented to study the effectiveness of the slotted screens to mitigate the wave-induced forces acting on the floating porous breakwater. For the computational calculations, the physical parameters are considered as follows: $h=$ $10 m, d_{1}=\frac{h}{3}, d_{2}=\frac{h}{5}, B=2 h w=\frac{h}{2}, s=1.0, f=1.0, \mu=0.5, \epsilon=0.1, C_{j}=h / 10$, $\tau_{1}=\tau_{2}=0.1$, unless otherwise mentioned.

In Fig. 4(a)-4(e), $C_{R}, C_{T}, C_{L}, F_{x}$, and $F_{z}$ are plotted as a function of the non-dimensional relative space between the slotted screen and the porous breakwater $w / L$ ( $L$ is the incident wavelength) for different porosities of the slotted screens. It is observed that $C_{R}$, $C_{T}, C_{L}, F_{x}$, and $F_{z}$ follow a periodic oscillatory pattern as the gap between the slotted screen and the floating porous breakwater increases. This periodic nature is due to continuous reflection and transmission by the slotted screens and porous breakwater. Fig. 4(a) shows that $C_{R}$ decreases with an increase in the porosity of the slotted screens. This is due to a large amount of incident wave energy being dissipated by the slotted screens and a porous floating breakwater, as seen in Fig. 4(c). Moreover, there is a leftward shift in the maximum of the reflection coefficient, and this is due to the phase shift of reflected waves by the slotted screens and the breakwater. In Fig. 4(b), $C_{T}$ increases with an increase in the porosity of the screens. It is observed that more than $98 \%$ of the wave energy dissipated with $20 \%$ porosity of slotted screens and $10 \%$ porosity of the porous breakwater. Further, Fig. 4(d) and 4(e) shows that the non-dimensional horizontal force $F_{x}$ and vertical force $F_{z}$ decrease with an increase in the geometrical porosity of the slotted screen. The reason is that a significant portion of the incoming wave energy is dissipated by the breakwater and slotted screens.

In both Fig. 4(d) and 4(e), the maximum wave-induced forces are observed when $\frac{w}{l} \approx \frac{n}{2}$, $n=1,2,3, \ldots$, and the minimum wave-induced forces acting on the porous breakwater are observed in the intermediate points of $\frac{(n-1)}{2}<\frac{w}{L}<\frac{n}{2}, n=1,2,3, \ldots$ for different porosities of the slotted screens.

In Fig. 5(a)-5(e), $C_{R}, C_{T}, C_{L}, F_{x}$, and $F_{z}$ are plotted as a function of the non-dimensional relative space between the slotted screen and the porous breakwater $\frac{w}{L}$ ( $L$ is the incident wavelength) for different submergence draft of the slotted screens $\frac{d_{1}}{h}$ with $\tau_{1}=\tau_{2}=0.1$. It 


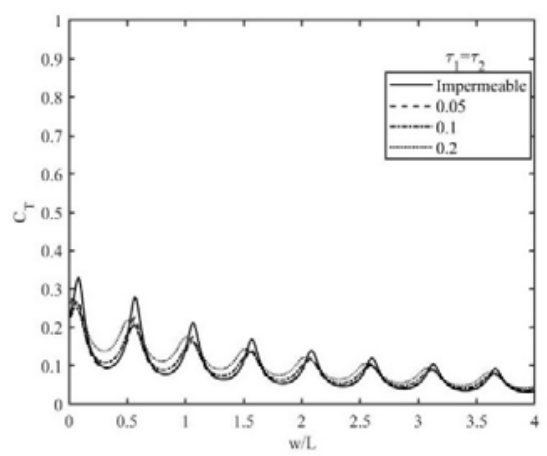

(a)

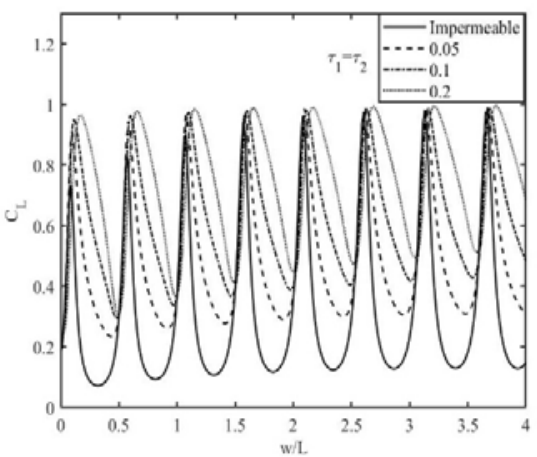

(c)

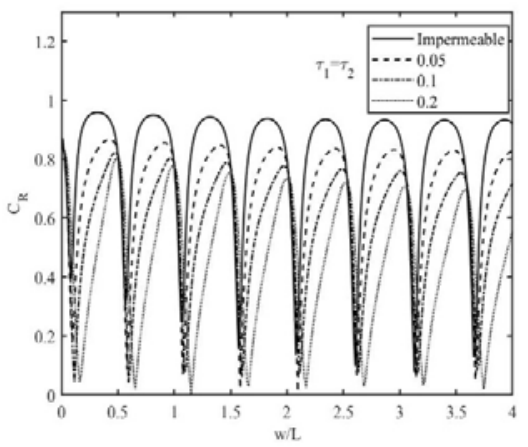

(b)

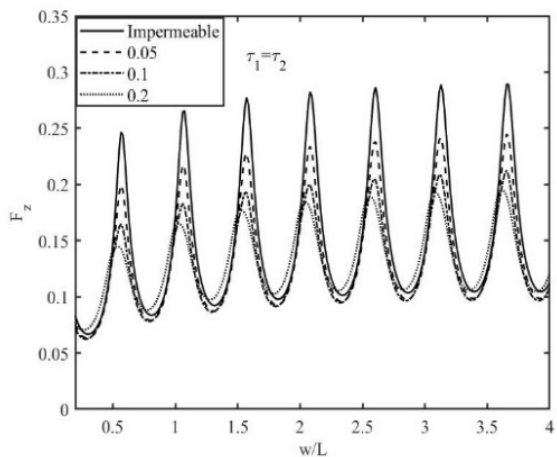

(d)

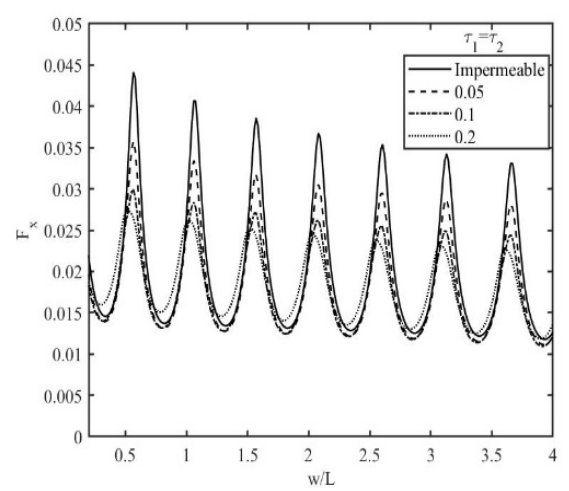

(e)

Figure 4: Variations of (a) $C_{R}$; (b) $C_{T}$; (c) $C_{L}$; (d) $F_{x}$; and (e) $F_{z}$ vs $w / L$ for different porosity of the slotted screens with $\tau_{1}=\tau_{2}, k_{0} h=2.0$.

is observed that $C_{R}, C_{T}, C_{L}, F_{x}$, and $F_{z}$ follow a periodic oscillatory pattern as the gap between the slotted screen and the floating porous breakwater increases. It is seen that in Fig. 5(a) and 5(b), the optimum $C_{R}$ and $C_{T}$ depend on the submergence draft of the surface-piercing slotted screens. However, the position of optimum does not depend on the submergence draft of the 


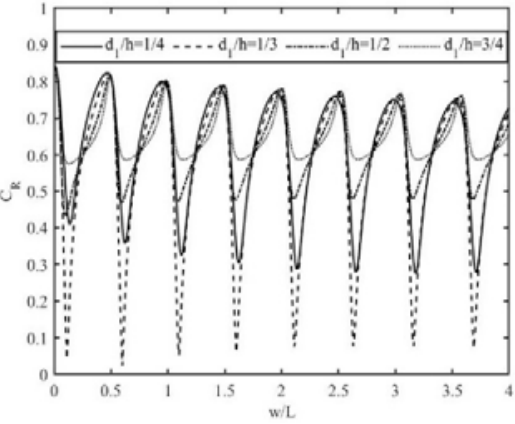

(a)

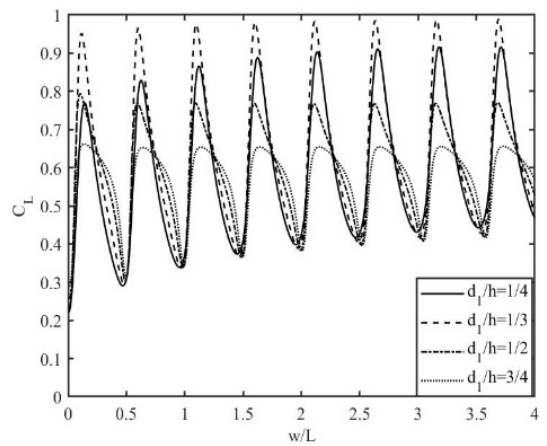

(c)

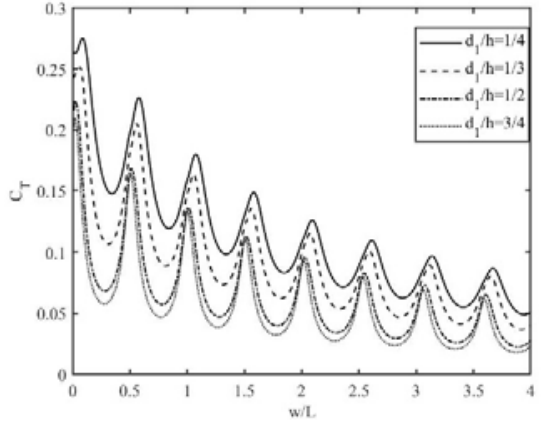

(b)

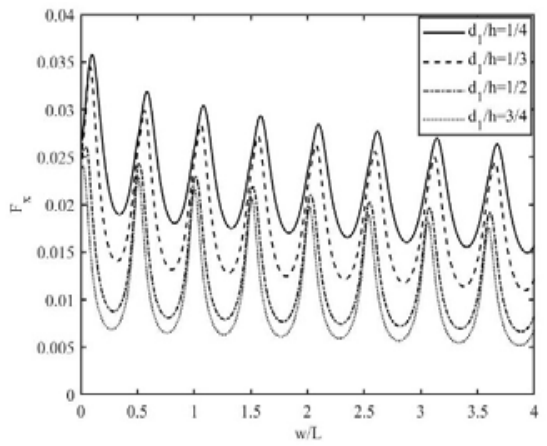

(d)

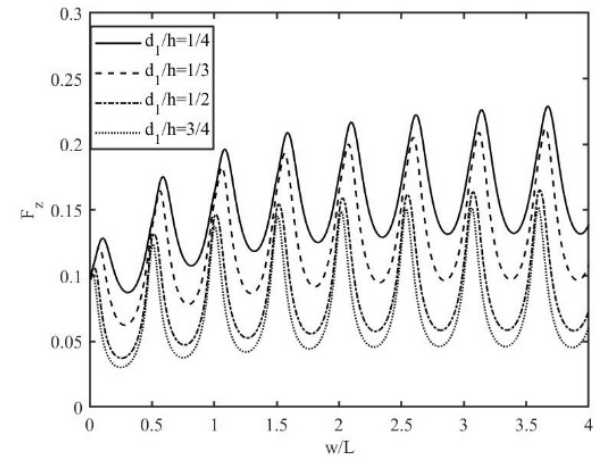

(e)

Figure 5: Variations of (a) $C_{R}$; (b) $C_{T}$; (c) $C_{L}$; (d) $F_{x}$; and (e) $F_{z}$ vs $w / L$ for different submergence draft of the slotted screens $\frac{d_{1}}{h}$ with $\tau_{1}=\tau_{2}=0.1, k_{0} h=2.0$.

surface-piercing slotted screens. In general, more amount of wave energy is concentrated at the free-surface. Therefore, surface-piercing slotted screens of shorter lengths dissipate more amount of wave energy. It is seen that for the present study, a slotted screen of submergence draft $\frac{d_{1}}{h}=\frac{1}{3}$ dissipates the maximum amount of incident wave energy with $10 \%$ screen porosity. The maximum and minimum wave-induced horizontal and vertical forces on the 
floating breakwater depend on the submergence draft of the slotted screen. However, the position of optimum does not depend on the submergence draft of the surface-piercing slotted screen. It is observed that the optimum positions are the same as that of Fig. 4(d) and 4(e) with small rightward phase shifts in the maximum wave forces.

\section{CONCLUSIONS}

This study develops an iterative-BEM-based numerical solution to study the effectiveness of slotted screens to mitigate the wave-induced hydrodynamic forces acting on the floating porous breakwater. The physical problem is studied within the framework of linear water wave theory. Further, the numerical results are validated with previous works as a limiting case of the present model. The numerical results show that

- The reflection, transmission, wave energy dissipation coefficients, and horizontal and vertical wave forces on the floating structure follow a periodic oscillatory pattern as the gap between the slotted screen and the floating porous breakwater increases.

- The reflection coefficient decreases with an increase in the porosity of the slotted screen. Further, more than $98 \%$ of the incoming wave energy can be dissipated with $20 \%$ porosities of the slotted screens and $10 \%$ porosity of the porous breakwater.

- The maximum wave-induced forces are observed when $\frac{w}{l} \approx \frac{n}{2}, n=1,2,3, \ldots$, and the minimum wave-induced forces acting on the porous breakwater are observed in the intermediate points $\frac{(n-1)}{2}<\frac{w}{L}<\frac{n}{2}, n=1,2,3, \ldots$ for different porosities of the slotted screen.

- A slotted screen of submergence length $\frac{d_{1}}{h}=\frac{1}{3}$ dissipates the maximum amount of incident wave energy with $10 \%$ porosity of the slotted screen.

- The optimum wave-induced horizontal and vertical forces on the floating breakwater depend on the submergence draft of the slotted screen. However, the positions of optima do not depend on the submergence draft of the surface-piercing slotted screen.

The present study can be extended to deal with the seabed undulations as well as irregular water waves. Further, the current solution method can be extended to various structures having complex geometries that arise in ocean engineering.

\section{ACKNOWLEDGEMENTS}

SK acknowledges the financial support received through the DST Project: DST/INSPIRE/04/2017/002460 to pursue this research work. Further, supports were received from BITS - Pilani, Hyderabad Campus through BITS/GAU/RIG/2019/H0631 and BITS/GAU/ACRG/2019/H0631(Additional Competitive Research Grant) projects. KPR acknowledges BITS - Pilani, Hyderabad Campus, for providing the financial support to pursue this research work.

\section{REFERENCES}

[1] Wang, C.M., Tay, Z.Y., Takagi, K. \& Utsunomiya, T., Literature review of methods for mitigating hydroelastic response of VLFS under wave action. Applied Mechanics Reviews, 63(3), 2010.

[2] Hong, D.C., Hong, S.Y. \& Hong, S.W., Numerical study of the motions and drift force of a floating OWC device. Ocean Engineering, 31(2), pp. 139-164, 2004. 
[3] Tay, Z.Y., Wang, C.M. \& Utsunomiya, T., Hydroelastic responses and interactions of floating fuel storage modules placed side-by-side with floating breakwaters. Marine Structures, 22(3), pp. 633-658, 2009.

[4] Nguyen, H.P., Dai, J., Wang, C.M., Ang, K.K. \& Luong, V.H., Reducing hydroelastic responses of pontoon-type VLFS using vertical elastic mooring lines. Marine Structures, 59, pp. 251-270, 2018.

[5] Ohta, K., Effect of attachment of a horizontal/vertical plate on the wave response of a VLFS. Proceedings of the 3rd International Workshop on Very Large Floating Structures, VLFS'99, vol. 1, ed. R. Cengiz Ertekin, Honolulu, Hawaii, USA, Proceedings Paper P1999-5, 1999.

[6] Watanabe, E., Utsunomiya, T., Kuramoto, M., Ohta, H., Torii, T. \& Hayashi, N., Wave response analysis of VLFS with an attached submerged plate. International Journal of Offshore and Polar Engineering, 13(3), 2003.

[7] Cheng, Y., Ji, C., Zhai, G. \& Oleg, G., Dual inclined perforated anti-motion plates for mitigating hydroelastic response of a VLFS under wave action. Ocean Engineering, 121, pp. 572-591, 2016.

[8] Wang, C.M. et al., Minimizing differential deflection in a pontoon-type, very large floating structure via gill cells. Marine Structures, 19(1), pp. 70-82, 2006.

[9] Wang, C.M., Riyansyah, M. \& Choo, Y.S., Reducing hydroelastic response of interconnected floating beams using semi-rigid connections. International Conference on Offshore Mechanics and Arctic Engineering, 43444, pp. 1419-1425, 2009.

[10] Gao, R.P., Tay, Z.Y., Wang, C.M. \& Koh, C.G., Hydroelastic response of very large floating structure with a flexible line connection. Ocean Engineering, 38(17-18), pp. 1957-1966, 2011.

[11] Hong, D.C., Hong, S.Y. \& Hong, S.W., Reduction of hydroelastic responses of a verylong floating structure by a floating oscillating-water-column breakwater system. Ocean Engineering, 33(5-6), pp. 610-634, 2006.

[12] Huang, Z., Li, Y. \& Liu, Y., Hydraulic performance and wave loadings of perforated/slotted coastal structures: A review. Ocean Engineering, 38(10), pp. 10311053, 2011.

[13] Vijay, K.G., Sahoo, T. \& Datta, R., Wave-induced responses of a floating structure near a wall in the presence of permeable plates. Coastal Engineering Journal, 62(1), pp. 35-52, 2020.

[14] Sun, W.Y., Qu, K., Kraatz, S., Deng, B. \& Jiang, C.B., Numerical investigation on performance of submerged porous breakwater to mitigate hydrodynamic loads of coastal bridge deck under solitary wave. Ocean Engineering, 213, p. 107660, 2020.

[15] Qiao, W., Wang, K.H., Duan, W. \& Sun, Y., Analytical model of wave loads and motion responses for a floating breakwater system with attached dual porous side walls. Mathematical Problems in Engineering, 2018.

[16] Yip, T.L. \& Chwang, A.T., Perforated wall breakwater with internal horizontal plate. Journal of Engineering Mechanics, 126(5), pp. 533-538, 2000.

[17] Liu, Y. \& Li, H.J., Iterative multi-domain BEM solution for water wave reflection by perforated caisson breakwaters. Engineering Analysis with Boundary Elements, 77, pp. 70-80, 2017.

[18] Sollitt, C.K. \& Cross, R.H., Wave transmission through permeable breakwaters. Coastal Engineering, 1972, pp. 1827-1846, 1973.

[19] Zhu, S. \& Chwang, A.T., Investigations on the reflection behaviour of a slotted seawall. Coastal Engineering, 43(2), pp. 93-104, 2001. 
[20] Koley, S., Panduranga, K., Almashan, N., Neelamani, S. \& Al-Ragum, A., Numerical and experimental modeling of water wave interaction with rubble mound offshore porous breakwaters. Ocean Engineering, 218, p. 108218, 2020.

[21] Li, A.J., Li, H.J. \& Liu, Y., Analytical study of oblique wave scattering by a submerged pile-rock breakwater. Proceedings of the Institution of Mechanical Engineers, Part M: Journal of Engineering for the Maritime Environment, 233(1), pp. 41-54, 2019.

[22] Koley, S. \& Panduranga, K., Energy balance relations for flow through thick porous structures. International Journal of Computational Methods and Experimental Measurements, 9(1), pp. 28-37, 2021.

[23] Hu, J., Zhao, Y. \& Liu, P.L.F., A model for obliquely incident wave interacting with a multi-layered object. Applied Ocean Research, 87, pp. 211-222, 2019. 